\title{
Protective Role of Nrf2 in Age-Related Hearing Loss and Gentamicin Ototoxicity
}

Tomofumi Hoshino', Keiji Tabuchi ${ }^{1,6}$, Bungo Nishimura' ${ }^{1}$, Shuho Tanaka ${ }^{1}$, Masahiro Nakayama ${ }^{1}$, Tetsuro Ishii ${ }^{2}$, Eiji Warabi ${ }^{2}$, Toru Yanagawa ${ }^{3}$, Ritsuku Shimizu ${ }^{4}$, Masayuki Yamamoto ${ }^{5}$, and Akira Hara ${ }^{1}$

1. Department of Otolaryngology,

2. Institute of Community Medicine,

3. Department of Oral and Maxillofacial Surgery,

Graduate School of Comprehensive Human Sciences, University of Tsukuba, Tsukuba, Japan

4. Department of Molecular Hematology,

5. Department of Medical Biochemistry, Tohoku University Graduate School of Medicine, Sendai, Japan

6. Corresponding author

Author's email: tomofumi@md.tsukuba.ac.jp

Corresponding author's address: ktabuchi@md.tsukuba.ac.jp

Keiji Tabuchi

Department of Otolaryngology

Graduate School of Comprehensive Human Sciences

University of Tsukuba

1-1-1 Tennodai, Tsukuba 305-8575, Japan

TEL: +81-298-53-3147

FAX: $+81-298-53-3147$

Number of pages: 26

Number of figures \& tables: 4 figures and no tables 


\section{Abstract}

Expression of antioxidant enzymes is regulated by transcription factor NF-E2-related factor (Nrf2) and induced by oxidative stress. Reactive oxygen species contribute to the formation of several types of cochlear injuries, including age-related hearing loss and gentamicin ototoxicity. In this study, we examined the roles of Nrf2 in age-related hearing loss and gentamicin ototoxicity by measuring auditory brainstem response thresholds in Nrf2-knockout mice. Although Nrf2-knockout mice maintained normal auditory thresholds at 3 months of age, their hearing ability was significantly more impaired than that of age-matched wild-type mice at 6 and 11 months of age. Additionally, the numbers of hair cells and spiral ganglion cells were remarkably reduced in Nrf2-knockout mice at 11 months of age. To examine the importance of Nrf2 in protecting against gentamicin-induced ototoxicity, 3-day-old mouse organ of Corti explants were cultured with gentamicin. Hair cell loss caused by gentamicin treatment was enhanced in the Nrf2-deficient tissues. Furthermore, the expressions of some Nrf2-target genes were activated by gentamicin treatment in wild-type mice but not in Nrf2-knockout mice. The present findings indicate that Nrf2 protects the inner ear against age-related hearing injuries and gentamicin ototoxicity by up-regulating antioxidant enzymes and detoxifying proteins. 


\section{Keywords}

NF-E2-related factor (Nrf2); reactive oxygen species (ROS); cochlea; age-related

hearing loss $(A H L)$; gentamicin ototoxicity 


\section{Introduction}

The antioxidant responsive element (ARE) is a cis-acting regulatory element, through which Nrf2 regulates transcription of genes encoding phase II detoxification enzymes, antioxidants, and other factors essential for cell survival. Under normal conditions, Nrf2 is anchored in the cytoplasm through interaction with Kelch-like ECH-associated protein 1 (Keap1) and subsequently proteolyzed by proteasomes. In contrast, under oxidative stress conditions, Keap1-censored electrophiles inhibit the proteolysis of Nrf2. Having thus escaped Keap1-mediated proteolysis, Nrf2 accumulates in the nucleus and activates ARE-mediated gene transcription $[1,2]$. To date, many genes driven by Nrf2, including heme oxygenase 1 (HO1), $\mathrm{NAD}(\mathrm{P}) \mathrm{H}$ :quinone oxidoreductase 1 (NQO1), NHR:quinone oxidoreductase 2 (NQO2), glutathione peroxidase (GPx), superoxide dismutase 1 (SOD1), and peroxiredoxin I (Prxl), have been reported to be involved in the antioxidant defense system [3-5].

Accumulated evidence suggests that reactive oxygen species (ROS) are involved in the pathogenesis of a wide range of cochlear injuries, including cochlear ischemia-reperfusion injury [6-9], acoustic injury [10-12], aminoglycoside ototoxicity [13], and age-related hearing loss (AHL) [14]. Because ROS are one of the Nrf2-inducing stressors, Nrf2-ARE responses are predicted to give rise to cochlear injuries. However, 
little is known about the protective role of Nrf2 in the cochlear pathophysiology.

In contrast, several contributions of Nrf2-driven antioxidant enzymes to the cochlea have been revealed. For example, SOD1 is one of the best-characterized enzymes in the cochlear pathology. Age-related hearing loss was accelerated in SOD1-knockout (KO) mice $[15,16]$, and SOD1-KO mice are more susceptible to noise-induced hearing loss [17]. Acoustic injury was also reported to be prevented by tempol, a SOD-mimetic agent [11]. Furthermore, $\mathrm{HO} 1$ and GPx effectively work to prevent inner ear injury after exposure to oxidative stresses [18-21]. Since Prxl scavenges hydrogen peroxide, lipid hydroperoxide, and peroxynitrite, Prxl is thought to contribute to the prevention of oxidative injury in the cochlea.

Here, we examined the contribution of Nrf2 to cochlear protection by using Nrf2-KO mice. The results from the auditory brainstem response (ABR) and morphological analyses indicated significant impairment of auditory function in aged mice in the absence of Nrf2. Furthermore, the cochlear cells of Nrf2-KO mice were severely injured after gentamicin treatment, to the extent that they lacked the expression of several Nrf2-driven antioxidant enzymes. These analyses of Nrf2-KO mice provide new insight into preventive medical procedures for age-related and drug-induced hearing loss. 


\section{Materials and Methods}

\section{Animals}

Nrf2-KO mice were produced and maintained on a BDF1 background as previously described [2]. Wild-type BDF1 mice were used as the control. The animals were maintained under a normal day/night cycle and given free access to food and water. The care and use of animals were approved by the Animal Research Committee and Safety Committee for Gene Recombination Research of the University of Tsukuba.

\section{ABR testing}

Mice were anesthetized with an intraperitoneal injection of pentobarbital sodium (Nembutal; Abbott Laboratories, Chicago, IL). Anesthesia was supplemented during the course of measurement of ABR as necessary.

Positive, negative, and ground electrodes were subcutaneously inserted at the vertex, mastoid, and back, respectively $[11,12,22]$. Bursts of 4,8 , or $16 \mathrm{kHz}$ pure tones were used to evoke ABR. Evoked responses were amplified, filtered with a bandpass of $200 \mathrm{~Hz}$ to $3 \mathrm{kHz}$, and averaged with 500 sweeps using a signal processor (Synax2100; NEC, Tokyo, Japan). The visual detection threshold was determined with increment or decrement of sound pressure at $5 \mathrm{~dB}$ steps. 


\section{Staining of hair cells}

Eleven-month-old mice were transcardially perfused with $4 \%$ paraformaldehyde under deep anesthesia with pentobarbital sodium. After decapitation, the cochleae were kept in the same fixative at $4^{\circ} \mathrm{C}$ overnight, decalcified with ethylenediaminetetraacetic acid (EDTA), and then dissected for surface preparation. Whole mounts of the organ of Corti were permeabilized with 5\% TritonX-100 (Sigma, St Louis, MO) in phosphate-buffered saline (PBS) with 10\% fetal bovine serum (FBS) for 10 minutes. The specimens were stained with a rhodamine-phalloidin probe $(1: 100$; Invitrogen, Carlsbad, CA) at room temperature for 1 hour [23]. Phalloidin specifically binds to cellular F-actin and is used to visualize the stereociliary arrays and cuticular plate of hair cells.

\section{Assessment of hair cell damage}

A hair cell was characterized as "missing" if neither the stereocilia nor the cuticular plate were observed in the cell by phalloidin staining. Quantitative results were obtained by evaluating 30 outer hair cells associated with 10 inner hair cells in a given microscopic field. The average of 5 separate counts was used to represent each 
specimen $[23,24]$.

\section{Assessment of spiral ganglion cells}

The Nrf2-KO and wild-type mice were killed at 11 months of age. The cochleae were fixed with $4 \%$ paraformaldehyde, decalcified with EDTA, and then embedded in paraffin. The cochleae were cut into 5 - $\mu$ m-thick midmodiolar sections. After the sections were stained with hematoxylin and eosin, the number of spiral ganglion cells was counted.

\section{Culture techniques}

The basal turn of the organ of Corti was dissected from the mice on postnatal day 3 (P3). The methods for culture of the organ of Corti were previously reported [23]. The explants were maintained in Dulbecco's modified Eagles medium (DMEM) with 10\% FBS, $25 \mathrm{mM}$ 4-(2-hydroxyethyl)-1-piperazineethanesulfonic acid, and $30 \mathrm{U} / \mathrm{mL}$ penicillin and cultured in an incubator at $37^{\circ} \mathrm{C}$ with $5 \% \mathrm{CO} 2$ and $95 \%$ humidity with or without gentamicin treatment at a concentration of $50 \mu \mathrm{M}$. For the morphological analysis, after 72 hours of culture, the explants were fixed with $4 \%$ paraformaldehyde in PBS for 20 minutes and then permeabilized with 5\% TritonX-100 in PBS with 10\% FBS for 10 
minutes $[23,24]$. The specimens were stained with a rhodamine-phalloidin probe, as described above.

\section{$R T-P C R$}

Dissected cochleae of P3-5 pups were cultured in DMEM media as described above for up to 24 hours. Total RNAs were isolated from the culture specimens using an Isogen RNA preparation kit (Nippon Gene, Tokyo, Japan). The cDNA samples were synthesized with SuperScript II reverse transcriptase (Invitrogen, Carlsbad, CA). qPCR Mastermix (Nippon Gene, Tokyo, Japan) was used for the analyses of the HO1 and NQO1 genes and a Power SYBR Green RNA-to-CT 1 step kit (Applied Biosystems, Carlsbad, CA) for the SOD1 and Prxl genes according to the manufacturer's instructions. Real-time RT-PCR was performed using an ABI-PRISM 7700 Sequence Detector System (Applied Biosystems, Carlsbad, CA). The primers were as follows, and otherwise were described previously [25]:

(SOD1 forward: 5'-GACAAACCTGAGCCCTAAG-3'; SOD1 reverse: 5'-CGACCTTGCTCCTTATTG-3'; Prxl forward: 5'-CGTTCTCACGGCTCTTTCTGT3'; PrxI reverse :5'-GCATTTCCTGAAGACATCTTGCT-3'). 


\section{Statistical analyses}

All data were evaluated by the $t$ test or two-way ANOVA. Any test resulting in a $p$ value of less than 0.05 was considered significant. Error bars represent the standard error of means. 


\section{Results}

\section{Hearing ability deteriorates rapidly with increasing age in Nrf2-KO mice.}

To elucidate the contribution of Nrf2 to the auditory organ, we examined hearing thresholds in Nrf2-KO mice by measuring the ABR thresholds. The ABR thresholds in the Nrf2-KO and age-matched wild-type mice at 3 months of age were not significantly different (Fig. 1). Next, to elucidate age-induced changes in hearing ability, we measured the ABR thresholds of the mice at 6 and 11 months of age. Expectedly, the ABR thresholds of the wild-type mice were elevated in an age-dependent manner (Fig. 1). Notably, the ABR thresholds of the 6- and 11-month-old Nrf2-KO mice rose to much higher levels than those of the age-matched wild-type mice (Fig. 1). These results indicated that Nrf2 was involved in maintaining hearing function in older mice.

Hair cells and spiral ganglion cells are missing in aged Nrf2-KO mice.

To elucidate the reason for the hearing disturbance in older Nrf2-KO mice, the cochlear morphology was microscopically examined and phalloidin staining used to observe the hair cells. As shown in Figure 2A, degeneration of the outer hair cells was apparent in the first cochlear region, while hair cell loss in the second turn and the region of inner hair cells was not obvious in the 11-month-old wild-type mice (Fig. 2A), 
consistent with previous reports. In contrast, the cochlear architecture of the Nrf2-KO mice was destroyed in the outer hair cell layer, and the hair cell loss extended into the inner hair cell layer of the second turn (Figure 2A). We next attempted quantitative analyses of the hair cells. In good agreement with the morphological analyses, the number of residual outer hair cells was significantly more decreased in the Nrf2-KO mice than in the age-matched wild-type mice ( $p<0.001$, two-way ANOVA;) (Fig. 2B, upper panel). In addition, significant inner hair cell loss in the first turn of the Nrf2-KO mouse cochlea was also observed ( $p<0.05$, two-way ANOVA) (Fig. 2B, lower panel).

Next, the ganglion cell population in the midmodiolar sections in the 11-month-old Nrf2-KO and wild-type mice was counted. Microphotographs of the lower first and lower second turns of these mice showed spiral ganglion cells in the Rosenthal canal (Fig. 3A). Loss of spiral ganglion cells in the lower turn of the cochlea was clearly observed in both the wild-type and the Nrf2-KO mice (Fig. 3A). On the other hand, spiral ganglion cell density in the upper turn was also decreased in the Nrf2-KO mice, whereas it was comparatively maintained in the wild-type mice (Fig. 3A). Quantitative analysis of the spiral ganglion cells in each turn revealed that the number of residual spiral ganglion cells was significantly more decreased in the Nrf2-KO mice than in the wild-type mice $(p<0.05$, two-way ANOVA). Taken together, hair cell loss and spiral ganglion cell loss 
rapidly progressed with age in Nrf2-KO mice, causing significant hearing impairments in older mice.

\section{Nrf2 knockout accelerated gentamicin ototoxicity.}

Nrf2 regulates expression of genes that protect cells from oxidative damage. To investigate the roles of Nrf2 in protection against oxidative stress in the cochlea, we attempted explant organ culture in the presence of gentamicin. Gentamicin is a well-known ototoxic drug, and its ototoxicity is involved in the ROS- and nitric oxide-related mechanisms.

Hair cell explants were dissected from P3 pups of Nrf2-KO and wild-type mice. Subsequently, the explants were incubated in $50 \mu \mathrm{M}$ gentamicin for 72 hours. As shown in Figure 4A, the outer hair cells were severely damaged in the Nrf2-KO mice. Quantitative analysis revealed that the number of residual outer hair cells was much more decreased in the Nrf2-KO mice than in the wild-type mice ( $p<0.01, t$ test) (Fig. 4B).

We next examined the expression of Nrf2-dependent enzymes in the cultured cochleae exposed to gentamicin. As shown in Figure 4C, the mRNA levels of HO1, NQO1, and SOD1 in the wild-type mice were increased at 6 hours and had recovered to the basal level at 24 hours after gentamicin exposure. On the other hand, such induction 
was not observed in the Nrf2-KO mice during the observation period. Especially, the expression of NQO1 and SOD1 genes after 6-hour exposure was significantly more elevated in the wild-type mice than in the Nrf2-KO mice ( $p<0.05, t$ test). In contrast, induction by gentamicin of $\operatorname{Prx} /$ gene expression was not detected in either the wild-type mice or the Nrf2-KO mice during this experiment. These findings suggest that Nrf2 protects the cochlear from gentamicin ototoxicity through induction of antioxidant enzymes. 


\section{Discussion}

Reactive oxygen species are involved in a wide range of cochlear injuries [6-14]. In addition, AHL is ascribed to the production of ROS, at least in part [26]. Nrf2 is believed to protect against oxidative tissue damage through ARE-mediated transcriptional activation of several phase II detoxifying enzymes and antioxidant enzymes. However, little is known about the function of Nrf2 in the cochlea. So et al. demonstrated the roles of $\mathrm{Nrf2}$ in the protection of cisplatin-induced hair cell loss via $\mathrm{HO}$ gene activation by means of an ex vivo organ culture assay [20]. Furthermore, recent work using mice suffering from progressive hearing loss demonstrated that sulforaphane, an Nrf2 inducer, was effective in alleviating the mouse phenotype [27]. Hence, the molecular dissection of the Nrf2 function in the cochlea has become very important for the development of preventive strategies for patients at high risk of ear damage. In this study, we clarified the importance of Nrf2 in the cochlea in protecting against AHL and ototoxic agents by using genetically manipulated Nrf2-KO mice.

The spiral shape of the cochlea was not disturbed in Nrf2-KO mice. In addition, the hearing ability of these mice was within normal levels until 3 months of age. In contrast, the hearing ability of Nrf2-KO mice rapidly worsened with age in comparison with that of wild-type mice and was accompanied by significant loss of hair cells and spiral ganglion 
cells. Therefore, we surmise that Nrf2 is preventive against progression of $\mathrm{AHL}$, while loss of Nrf2 is dispensable during auditory organ development.

Aminoglycoside antibiotics, such as gentamicin, are ototoxic drugs damaging to cochlear hair cells. As in cisplatin-induced ototoxicity, ROS are one of the leading factors involved in aminoglycoside-induced cochlear injury. We here demonstrated that Nrf2 protects against progression of gentamicin-induced hair cell damage by regulating antioxidant enzymes. The expression of $N Q O 1$ and SOD1, which are representative ARE-dependent Nrf2-mediated detoxifying/antioxidant enzymes, was significantly upregulated after 6-hour gentamicin exposure in wild-type mice, but such upregulation was not observed in Nrf2-KO mice. We surmise that in a similar manner to these 2 genes, $\mathrm{HO} 1$ is cooperatively involved in otoprotection, since it is one of the important Nrf2-mediated enzymes against oxidative stress-induced inner ear injury. We could not detect any significant difference in $\mathrm{HO}$ gene induction by gentamicin exposure between the wild-type and Nrf2-KO mice, probably because the expression of $\mathrm{HO} 1$ is regulated by a combination of Nrf2 and Bach1, which is another b-Zip protein Bach1 [28]. Further analysis using Nrf2- and Bach1-double KO mice is required to clarify the regulation of $\mathrm{HO} 1$ in the cochlea by gentamicin exposure. It is noteworthy that the expression of such Nrf2-target genes was increased in a transient manner when the 
induction response was complete by 24 hours. This result is somewhat unexpected because the induction of Nrf2 target genes by conventional Nrf2-inducers usually continues beyond 24 hours. It was previously reported that a transient response by diethyl maleate, a strong Nrf2 inducer, was observed in HO1 but not in NQO1 gene expression because of a gene-specific epigenetic modification [29]. We speculate that a variety of modification mechanisms divers the gene regulation profiles depending on the tissue identity.

In conclusion, the present study demonstrated that disruption of Nrf2 causes AHL and renders the cochlea vulnerable to gentamicin ototoxicity. Although Nrf2 does not contribute to the formation of the inner ear in the developmental period, it cleans up the ROS induced by several postbirth environmental factors, thereby minimizing AHL-related cochlear injuries. We propose that compounds that activate Nrf2 prevent progression of cochlear injury related to $\mathrm{AHL}$ and ototoxic agents. 


\section{Acknowledgement}

This work was supported by a Grant-in-aid for Young Scientists (B) (22791567) from the Ministry of Education, Culture, Sports, Science, and Technology of Japan. 


\section{References}

[1] K. Itoh, T. Ishii, N. Wakabayashi, M. Yamamoto, Regulatory mechanisms of cellular response to oxidative stress, Free Radic. Res. 31 (1999) 319-324.

[2] K. Itoh, N. Wakabayashi, Y. Katoh, T. Ishii, T. O'Connor, M. Yamamoto, Keap1 regulates both cytoplasmic-nuclear shuttling and degradation of Nrf2 in response to electrophiles, Genes Cells 8 (2003) 379-391.

[3] T. Ishii, K. Itoh, S. Takahashi, H. Sato, T. Yanagawa, Y. Katoh, S. Bannai, M. Yamatomo, Transcriptional factor Nrf2 coordinately regulates a group of oxidative stress-inducible genes in macrophages, J. Biol. Chem. 275 (2000) 16023-16029.

[4] G. Schreibelt, J. van Horssen, S. van Rossum, C.D. Dijkstra, B. Drukarch, H.E. de Vries, Therapeutic potential and biological role of endogenous antioxidant enzymes in multiple sclerosis pathway, Brain Res. Rev. 56 (2007) 322-330.

[5] J.A. Johnson, D.A. Johnson, A.D. Kraft, M.J. Calkins, R. Jakel, M.R. Vargas, P.C. Chen, The Nrf2-ARE pathway. An indicator and modulator of oxidative stress in neurodegeneration, Ann. N. Y. Acad. Sci. 1147 (2008) 61-69.

[6] K. Tabuchi, Z. Ito, T. Wada, A. Hara, J. Kusakari, The effect of mannitol upon cochlear dysfunction induced by transient local anoxia, Hear. Res. 126 (1998) 28-36.

[7] K. Tabuchi, J. Kusakari, Z. Ito, K. Takahashi, T. Wada, A. Hara, Effect of nitric oxide 
synthase inhibitor on cochlear dysfunction induced by transient local anoxia, Acta. Otolaryngol. 119 (1999) 179-184.

[8] K. Tabuchi, H. Okubo, K. Fujihira, S. Tsuji, A. Hara, J. Kusakari, Protection of outer hair cells from reperfusion injury by an iron chelator and a nitric oxide synthase inhibitor in the guinea pig cochlea, Neurosci. Lett. 307 (2001) 29-32.

[9] K. Tabuchi, S. Tsuji, K. Fujihira, K. Oikawa, A. Hara, J. Kusakari, Outer hair cells functionally and structurally deteriorate during reperfusion, Hear. Res. 173 (2002) 153-163.

[10] T. Yamasoba, J. Schacht, F. Shoji, J.M. Miller, Attenuation of cochlear damage from noise trauma by an iron chelator, a free radical scavenger and glial cell line-derived neurotrophic factor in viro, Brain Res. 815 (1999) 317-325.

[11] H. Murashita, K. Tabuchi, T. Hoshino, S. Tsuji, A Hara, The effects of tempol, 3-aminobenzamide and nitric oxide synthase inhibitors on acoustic injury of the mouse cochlea, Hear. Res. 214 (2006) 1-6.

[12] T. Hoshino, K. Tabuchi, Y. Hirose, I. Uemaetomari, H. Murashita, T. Tobita, A. Hara, The non-steroidal anti-inflammatory drugs protect mouse cochlea against acoustic injury, Tohoku J. Exp. Med. 216 (2008) 53-59.

[13] Y.H. Choung, A. Taura, K. Pak,S.J. Choi, M. Masuda, A.F. Ryan, Generation of 
highly-reactive oxygen species is closely related to hair cell damage in rat organ of corti treated with gentamicin, Neuroscience 161 (2009) 214-226.

[14] C. Riva, E. Donadieu, J. Maqnan, J.P. Lavielle, Age-related hearing loss in CD/1 mice is associated to ROS formation and HIF target proteins up-regulation in the cochlea, Exp. Gerontol. 42 (2007) 327-336.

[15] S.L. McFadden, D.L. Ding, A.G. Reaume, D.G. Flood, R.J. Salvi, Age-related cochlear hair cell loss is enhanced in mice lacking copper/zinc superoxide dismutase, Neurobiol. Aging 20 (1999) 1-8.

[16] S.L. McFadden, D.L. Ding, R.F. Burkard, H. Jiang, A.G. Reaume, D.G. Flood, R.J. Salvi, Cu/Zn SOD deficiency potentiates hearing loss and cochlear pathology in aged 129,CD-1 mice, J. Comp. Neurol. 413 (1999) 101-112.

[17] K.K. Ohlemiller, S.L. McFadden, D.L. Ding, D.G. Flood, A.G. Reaume, E.K. Hoffman, R.W. Scott, J.S. Wright, G.V. Putcha, R.J. Salvi, Targeted deletion of the cytosolic $\mathrm{Cu} / \mathrm{Zn}$-superoxide dismutase gene (Sod1) increases susceptibility to noise-induced hearing loss, Audiol. Neurootol. 4 (1999) 237-246.

[18] D.A. Fairfield, A.C. Kanicki, M.I. Lomax, R.A. Altschuler, Induction of heat shock protein 32 (Hsp32) in the rat cochlea following hyperthermia, Hear. Res. 188 (2004) 1-11. 
[19] H.J. Kim, H.S. So, J.H. Lee, J.H. Lee, C. Park, S.Y. Park, Y.H. Kim, M.J. Youn, S.J. Kim, S.Y. Chung, K.M. Lee, R. Park, Heme oxygenase-1 attenuates the cisplatin-induced apoptosis of auditory cells via down-regulation of reactive oxygen species generation, Free Radic. Biol. Med. 40 (2006) 1810-1819.

[20] H.S. So, H.J. Kim, J.H. Lee, J.H. Lee, S.Y. Park, C. Park, Y.H. Kim., J.K. Kim, K.M. Lee, K.S. Kim, S.Y. Chung, W.C. Jang, S.K. Moon, H.T. Chung, R.K. Park, Flunarizine induces Nrf2-mediated transcriptional activation of heme oxygenase-1 in protection of auditory cells from cisplatin, Cell Death Differ. 13 (2006) 1763-1775.

[21] J. Kil, C. Pierce, H. Tran, R. Gu, E.D. Lynch, Ebselen treatment reduces noise induced hearing loss via the mimicry and induction of glutathione peroxidase, Hear. Res. 226 (2007) 44-51.

[22] K. Tabuchi, M. Suzuki, A. Mizuno, A. Hara, Hearing impairment in TRPV4 knockout mice, Neurosci. Lett. 382 (2005) 304-308.

[23] K. Tabuchi, K. Pak, E. Chavez, A.F, Ryan, Role of inhibitor of apoptosis protein in gentamicin-induced cochlear hair cell damage, Neuroscience 149 (2007) 213-222.

[24] W.H. Chung, K. Pak, B. Lin, N. Webster, A.F. Ryan, A PI3K pathway mediates hair cell survival and opposes gentamicin toxicity in neonatal rat organ of Corti, J. Assoc. Res. Otolaryngol. 7 (2006) 373-382. 
[25] T. Suzuki, V.P. Kelly, H. Motohashi, O. Nakajima, S. Takahashi, S. Nishimura, M. Yamamoto, Deletion of the selenocysteine tRNA gene in macrophages and liver results in compensatory gene induction of cytoprotective enzymes by Nrf2, J. Biol. Chem. 283 (2008) 2021-2030.

[26] X.Z. Liu, D. Yan, Ageing and hearing loss, J. Pathol. 211 (2007) 188-197.

[27] L. Kong, X. Zhou, J.F. McGinnis, F. Li, W. Cao, Molecular mechanisms underlying cochlear degeneration in the tubby mouse and the therapeutic effect of sulforaphane, Neurochem. Int. 54 (2008) 172-179.

[28] J. Sun, H, Hoshino, K. Takaku, O. Nakajima, A. Muto, H. Suzuki, S. Tashiro, S. Takahashi, S. Shibahara, J. Alam, M.M. Taketo, M. Yamamoto, K. Igarashi, Hemoprotein Bach1 regulates enhancer availability of hemo oxygenase-1 gene, EMBO J. 21 (2002) 5216-5224.

[29] J. Zhang, T. Ohta, A. Maruyama, T. Hosoya, K. Nishikawa, J.M. Maher, S. Shibahara, K. Itoh, M. Yamamoto, BRG1 interacts with Nrf2 to selectively mediate HO-1 induction in response to oxidative stress, Mol. Cell. Biol. 26 (2006) 7942-7952. 


\section{Legends}

Figure 1. ABR thresholds of Nrf2-KO and wild-type mice at 3, 6, and 11 months of age. The ABR thresholds in the Nrf2-KO mice at 6 and 11 months of age were significantly more elevated than those in the age-matched wild-type mice $(p<0.01$, two-way ANOVA), while the Nrf2-KO mice showed a similar ABR pattern to that of the wild-type mice at 3 months of age. Ten wild-type and 10 Nrf2-KO mice at 3 months of age, 10 wild-type and 6 Nrf2-KO mice at 6 months of age, and 10 wild-type and 12 Nrf2-KO mice at 11 months of age were used for this experiment.

Figure 2. Age-related degradation of cochlear hair cells in Nrf2-KO mice. (A) Representative sections through the organ of Corti showing inner and outer hair cells of wild-type and Nrf2-KO mice at 11 months of age. Bar: $50 \mu \mathrm{m}$. (B) Quantitative analyses of the number of residual outer and inner hair cells in mice at 11 months of age. Note the severe outer hair cell loss in the Nrf2-KO mice not only in the first but also in the second turn ( $p<0.01$, two-way ANOVA). The hair cell loss extended into the inner hair cell region in the first turn of the Nrf2-KO mice ( $p<0.01$, two-way ANOVA). Six or 7 mice in each group were used for this experiment. 
Figure 3. Age-related spiral ganglion cell loss in Nrf2-KO mice. (A) Representative sections through the organ of Corti showing spiral ganglion cells of the wild-type and Nrf2-KO mice at 11 months of age. The areas of the Rosenthal canal in the lower first turn (arrows) and the lower second turn (arrowheads) are indicated by dotted lines. Bar: upper panel $100 \mu \mathrm{m}$; lower panel $20 \mu \mathrm{m}$. (B) Quantitative analyses of the number of residual spiral ganglion cells. The residual spiral ganglion cell population was significantly more decreased in the Nrf2-KO mice than in the wild-type mice $(p<0.05$, two-way ANOVA). Five mice in each group were used for this experiment.

Figure 4. Gentamicin ototoxicity in the cultured cochlear hair cells. (A) Representative sections of hair cell explants exposed to gentamicin for 72 hours. The hair cells of the Nrf2-KO mice were more damaged than those of the wild-type mice. Bar: $50 \mu \mathrm{m}$. (B) Quantitative analysis of the number of residual outer hair cells after gentamicin exposure. The number of residual outer hair cells in the Nrf2-KO mice was significantly more reduced than that in the wild-type mice $(p<0.01, t$ test). Ten wild-type and 15 Nrf2-KO mice were used for this experiment. (C) Expression levels of genes coding for Nrf2-driven antioxidant enzymes after gentamicin exposure. The vertical axes indicate the relative ratio of the gene expression in the explants exposed to gentamicin to that in 
the untreated explants. Four wild-type and 4 Nrf2-KO mice were used for this experiment. 
Figure 2

Fig. 2
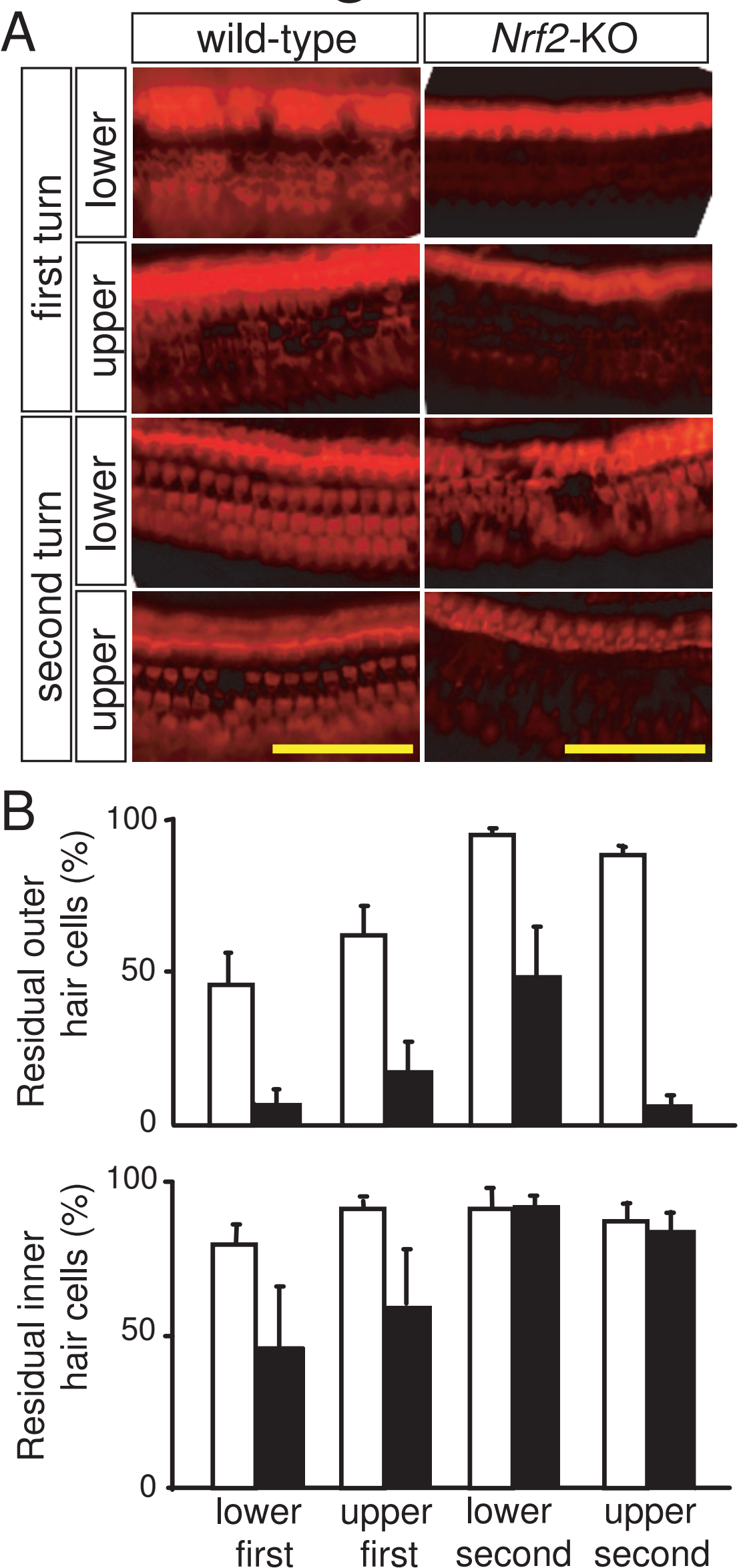
$\square$ wild-type
Nrf2-KO 
Figure 3

Fig. 3

$A$

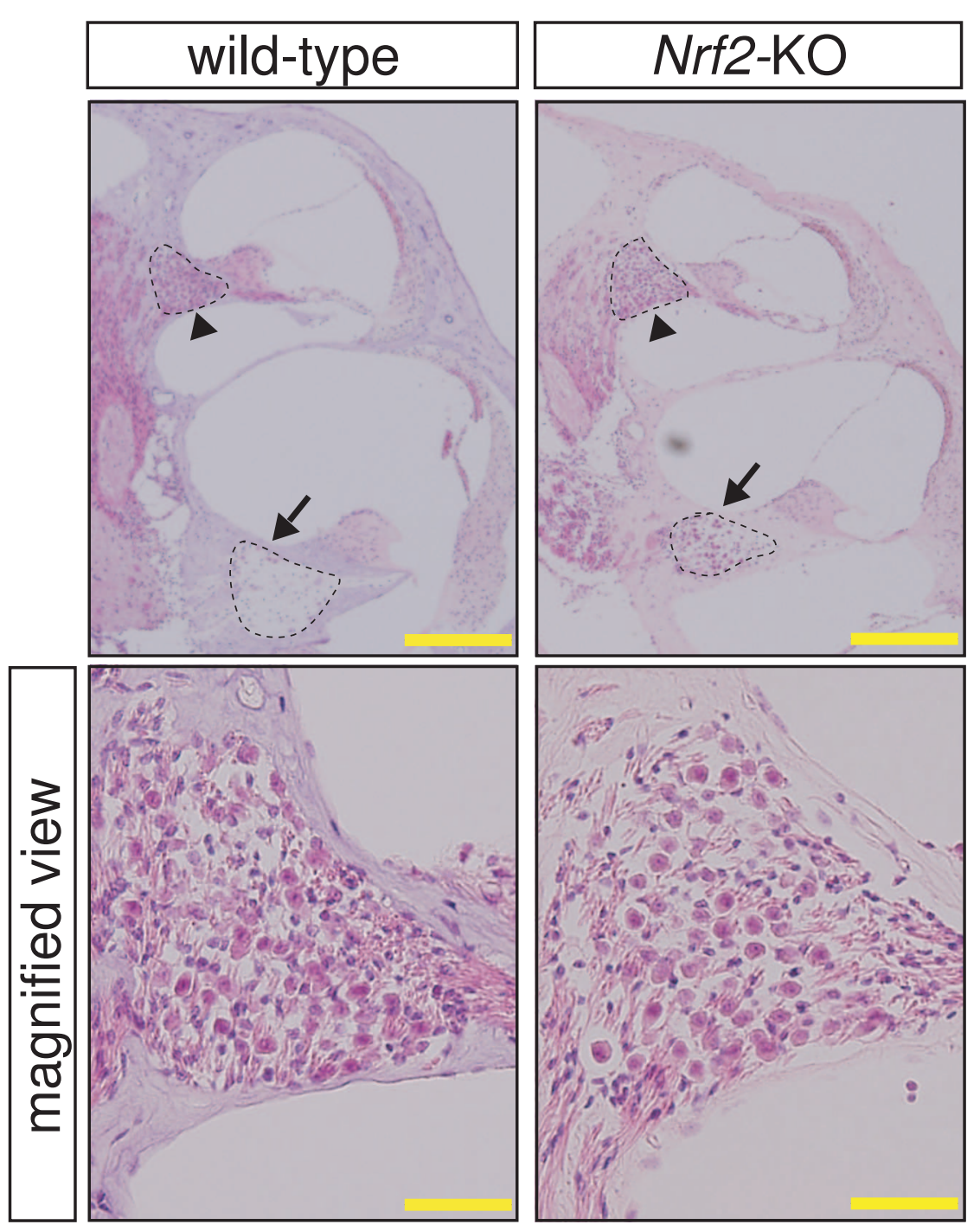

$\mathrm{B}$
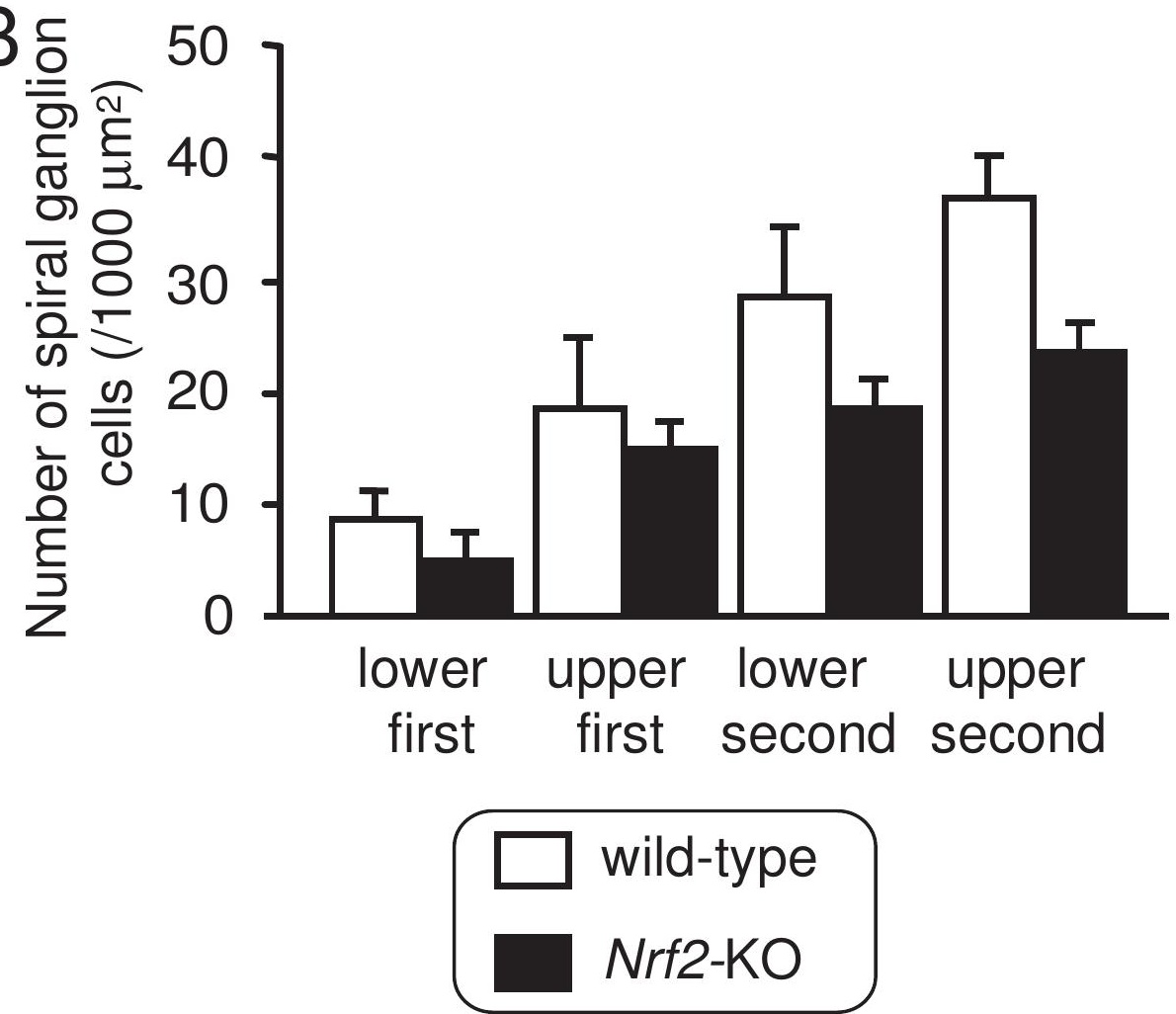


\section{Fig. 4}

A
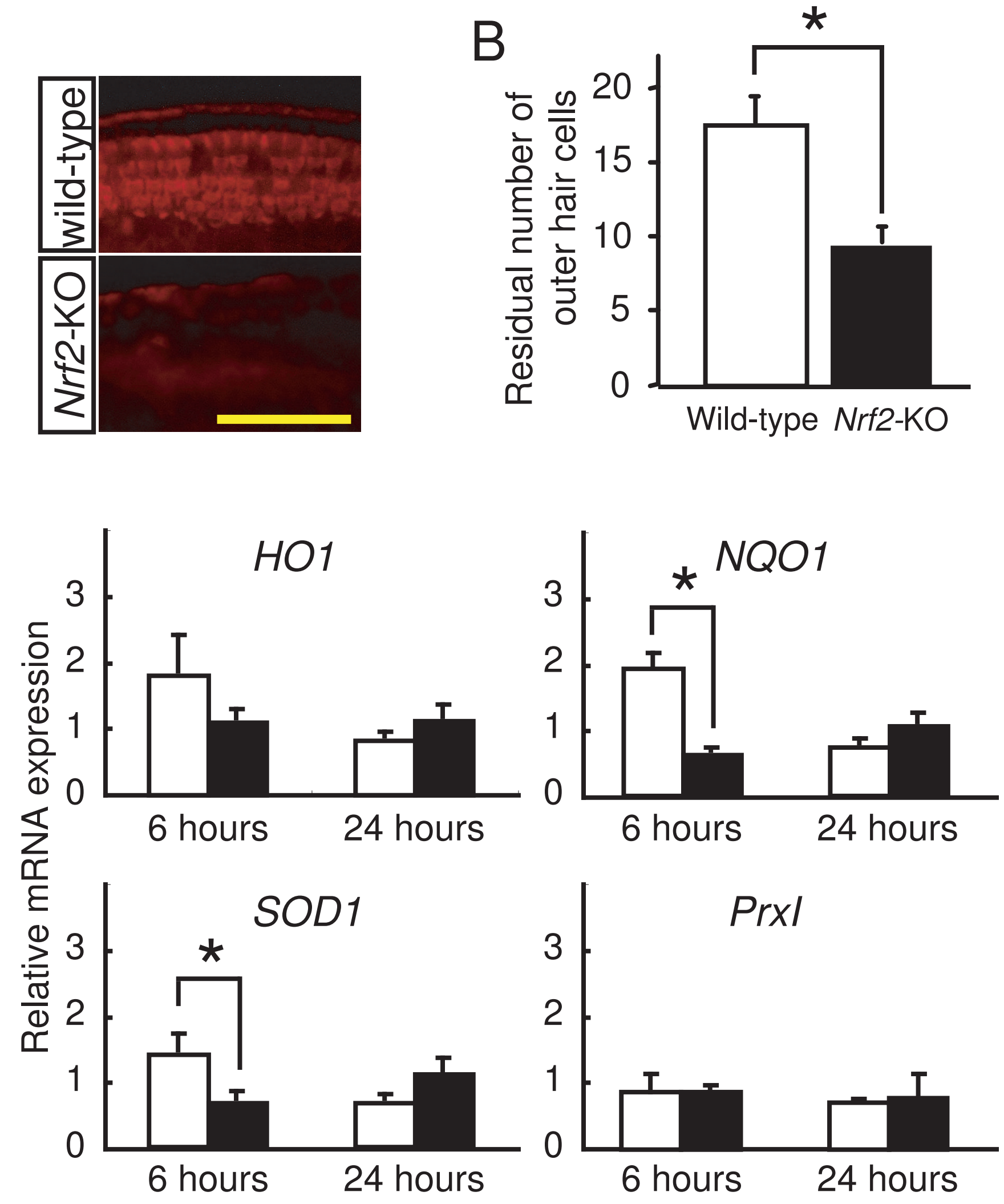

$\square$ wild-type

Nrf2-KO 